\title{
Evaluation of the structural quality of modeled proteins by using globularity criteria Susan Costantini ${ }^{\dagger 1,2,3}$, Angelo M Facchiano ${ }^{\dagger 1,3}$ and Giovanni Colonna*1,2
}

Address: ${ }^{1}$ CRISCEB (Research Center of Computational and Biotechnological Sciences), Second University of Naples, Via Costantinopoli 16, 80138 Naples, Italy, ${ }^{2}$ Department of Biochemistry and Biophysics, Second University of Naples, Via Costantinopoli 16, 80138 Naples, Italy and ${ }^{3}$ Laboratory of Bioinformatics and Computational Biology, Institute of Food Science, CNR, Via Roma 52 A/C, 83100 Avellino, Italy

Email: Susan Costantini - susan.costantini@isa.cnr.it; Angelo M Facchiano - angelo.facchiano@isa.cnr.it;

Giovanni Colonna* - giovanni.colonna@unina2.it

* Corresponding author †Equal contributors

Published: 9 March 2007

BMC Structural Biology 2007, 7:9 doi:10.1 186/1472-6807-7-9

This article is available from: http://www.biomedcentral.com/1472-6807/7/9

(C) 2007 Costantini et al; licensee BioMed Central Ltd.

This is an Open Access article distributed under the terms of the Creative Commons Attribution License (http://creativecommons.org/licenses/by/2.0), which permits unrestricted use, distribution, and reproduction in any medium, provided the original work is properly cited.
Received: 6 December 2006

Accepted: 9 March 2007

\begin{abstract}
Background: The knowledge of the three-dimensional structure of globular proteins is fundamental for a detailed investigation of their functional properties. Experimental methods are too slow for structure investigation on a large scale, while computational prediction methods offer alternatives that are continuously being improved. The international Comparative Assessment of Structure Prediction (CASP), an "a posteriori" evaluation of the quality of theoretical models when the experimental structure becomes available, demonstrates that predictions can be successful as well as unsuccessful, and this suggests the necessity for evaluations able to discard "a priori" the wrong models.

Results: We analyzed different structural properties of globular proteins for experimentally solved proteins belonging to the four different structural classes: "mainly alpha", "mainly beta", "alpha/beta" and "alpha+beta". The properties were found to be linearly correlated to protein molecular weight, but with some differences among the four classes. These results were applied to develop an evaluation test of theoretical models based on the expected globular properties of proteins. To verify the success of our test, we applied it to several protein models submitted to the sixth edition of CASP. The best theoretical models, as judged by CASP assessors, were in agreement with the expected properties, while most of the low-quality models had not passed our evaluations.

Conclusion: This study supports the need for careful checks to avoid the diffusion of incorrect structural models. Our test allows the evaluation of models in the absence of experimental reference structures, thereby preventing the diffusion of incorrect structural models and the formulation of incorrect functional hypotheses. It can be used to check the globularity of predicted models, and to supplement other methods already used to evaluate their quality.
\end{abstract}

\section{Background}

Globular proteins are critical players in the cell whose function is dictated by their characteristic structure. Because the number of proteins with known sequence far exceeds the number with known structure, the ability of computational methods to predict the structure from sequence is considered extremely valuable to investigate their functional properties [1]. Proper valuation of the 
quality of models is the basic problem of any computational approach to structure prediction. A basic difference between experimental and computational approaches to solve the $3 \mathrm{D}$ structure of proteins is that X-ray or NMR protocols start from high protein concentration conditions, while "ab initio" predictive methods run on a single protein molecule. This means that most predictive methods can not take into account the strong influence of environment on the globular structure of the protein. Fundamental features for determining the globularity are solubility, packing stability, folding, and compactness $[2,3]$. Therefore, to improve the quality of protein structure prediction, their effects should be simulated. As an alternative, at least to evaluate the quality of the predictions, it is necessary to know whether the structural properties typical of globular proteins are retained also by theoretical models.

In 1951 Pauling was the first to consider the importance of the intra-molecular H-bonds in protein structures, emphasizing their role in stabilizing the alpha-helices and beta-strands $[4,5]$. Many studies have pointed out that $\mathrm{H}$ bonds contribute favorably to globular protein stability [6]. Also important in understanding protein globularity is the packing of the protein atoms (i.e., the efficient filling of space). Protein cavities or packing defects occur with relative abundance, both within and between folding units, and the creation or filling of such cavities affects protein stability and structure, disturbing core packing $[7,8]$. Moreover, the variation of atom packing in a data set of globular proteins can be due to a complex combination of protein size and secondary structure, and amino acid composition [9]. These differences in protein packing are conserved in protein families of similar structure; the modeling of protein structures based on homologous templates should take into account the packing of the template structure. However, the prediction of protein models obtained by "ab-initio" methods is much more difficult, since there is no template structure as reference. In these cases, and in general for all predicted models, we should always control those parameters able to confirm that the obtained structures have the typical properties of globular proteins. A search of the scientific literature found only two methods that make predictions for globu- larity, and both are sequence-based rather than structurebased $[10,11]$.

Therefore, we first studied and analyzed a valuable set of experimental protein structures belonging to the four known structural classes ("mainly alpha", "mainly beta", "alpha/beta", and "alpha+beta") in terms of H-bonds, voids, solvent-accessible surface area, and water molecules in a layer of 5 Ångstroms. This analysis allowed us to deduce structural parameters useful in determining the protein globularity and to define operative criteria to evaluate models, particularly those predicted by "ab-initio" methods. These structural parameters were combined together as an index of globularity by which we tested thirteen sets of models submitted to CASP6 protein structure prediction experiment in the New Fold (NF) and difficult Fold Recognition Analogous (FR/A) categories.

\section{Results}

\section{Protein set selection}

Protein structures, solved by NMR or X-Ray crystallography with resolution of $2.5 \AA$ or better, were extracted from PDBselect [12]. These proteins were subdivided into four structural classes, i.e. "mainly alpha", "mainly beta", "alpha/beta" and "alpha+beta", on the bases of secondary structures assigned by DSSP program and of the SCOP database to classify the alpha-beta proteins as alpha+beta or alpha/beta [13-15]. Table 1 reports the number of structures comprised in each set and the mean ratios between the residue number in helix, in beta-strands and in "coil", and the total residue number. These structures were analyzed in terms of $\mathrm{H}$-bonds, accessible surface area, water molecules and voids, in order to define some typical parameters for each protein structural class.

\section{Hydrogen bonding}

The H-bonds in these protein structures were evaluated using the Hbplus package and classified as main-chain donor to main-chain acceptor (MM), main-chain donor to side-chain acceptor (MS), side-chain donor to mainchain acceptor (SM) and side-chain donor to side-chain acceptor (SS). The total number of $\mathrm{H}$-bonds ranged between 14 and 377 in "mainly-alpha", 9 and 145 in "mainly-beta", 15 and 351 in "alpha+beta" and 41 and

Table I: Training protein sets.

\begin{tabular}{lcccc}
\hline & Number of structures & helix/total & beta/total & Coil/total \\
\hline mainly-alpha & 124 & $0.648( \pm 0.12)$ & & $0.36( \pm 0.12)$ \\
mainly-beta & 81 & & $0.37( \pm 0.13)$ & $0.63( \pm 0.13)$ \\
alpha/beta & 75 & $0.38( \pm 0.08)$ & $0.21( \pm 0.04)$ & $0.41( \pm 0.07)$ \\
alpha+beta & 132 & $0.29( \pm 0.08)$ & $0.27( \pm 0.079$ & $0.44( \pm 0.09)$ \\
\hline
\end{tabular}

We report the number of structures in the four sets and the mean ratios between the residues in helix, in beta-strands and in "coil" and the total residue number. Standard deviations are reported in parentheses. 
400 in "alpha/beta" proteins. The correlation coefficients between the total number of H-bonds or MM-, MS-, SMand SS-type H-bonds are reported in Table 2. The total number of MM-type H-bonds increased linearly with the molecular weight of selected proteins belonging to all four protein sets (Figures 1 and Additional Files 1, 2, 3). The mean ratios between MM-, MS-, SM- or SS-type Hbonds and the total number of $\mathrm{H}$-bonds were evaluated and are reported in Table $3 \mathrm{~A}$.

\section{Accessible surface area}

For each selected structure the total accessible surface area was evaluated by summing the related polar and nonpolar components. The total area accessible to solvent increased in a linear way with the protein molecular weights (Figures 1 and Additional Files 1, 2, 3) with correlation coefficients higher than 0.9 in all four structural classes (Table 2). The mean ratios between non-polar accessibility value and the related total accessibility are reported in Table 3B.

\section{Water molecules}

A layer of $5 \AA$ around each protein was considered, and the number of water molecules and of H-bonds between the water molecules and the protein residues was evaluated. The number of water molecules increased linearly with the molecular weight of the proteins in all four sets with correlation coefficients higher than 0.9 (Table 2, Figures 1 and Additional Files 1, 2, 3).

\section{Void analysis}

The AVP program was run on the four protein sets using two different probes: the first to identify the holes in the interior of a protein (a zero-sized probe) and the second to delimit the solvent accessible regions on the surface (probe with a radius of 1.4 ). The total void volume is obtained summing the total buried and surface void volumes. This increased linearly with the protein size with correlation coefficients higher than 0.9 (see Table 2, Figures 1 and Additional Files 1, 2, 3), according to Fleming and Richards [9]. The mean ratios between the total surface and buried void volumes and the total void volume are reported in Table $3 \mathrm{C}$.

\section{Score value for training proteins}

By using the corresponding linear regression equations obtained for the structural parameters (see Additional File 4 ), we computed a score for each protein (see Methods for details). The score value increases if the protein properties are well outside the expected values. For each protein set, we determined the threshold (or cutoff) value as the score which includes $90 \%$ of the examined proteins. The threshold value was found to be 5.9 in the case of "mainlyalpha" and "mainly-beta" proteins, and 5.1 for "alpha/

Table 2: Correlation coefficients in the four structural classes.

\begin{tabular}{|c|c|c|c|c|}
\hline & mainly-alpha & mainly-beta & alpha/beta & alpha+beta \\
\hline \multicolumn{5}{|l|}{ H-bonds } \\
\hline MM-type & 0.95 & 0.83 & 0.97 & 0.96 \\
\hline MS-type & 0.61 & 0.49 & 0.83 & 0.79 \\
\hline SM-type & 0.70 & 0.39 & 0.85 & 0.81 \\
\hline SS-type & 0.67 & 0.41 & 0.82 & 0.81 \\
\hline Total & 0.93 & 0.80 & 0.97 & 0.95 \\
\hline \multicolumn{5}{|l|}{ Total Accessibility } \\
\hline polar- & 0.89 & 0.89 & 0.93 & 0.94 \\
\hline non polar- & 0.92 & 0.90 & 0.95 & 0.92 \\
\hline Total & 0.95 & 0.93 & 0.96 & 0.94 \\
\hline \multicolumn{5}{|l|}{ Void } \\
\hline total void volumes & 0.87 & 0.9 & 0.92 & 0.91 \\
\hline void numbers & 0.97 & 0.91 & 0.99 & 0.97 \\
\hline \multicolumn{5}{|l|}{ water } \\
\hline molecule number & 0.93 & 0.91 & 0.97 & 0.93 \\
\hline protein-water $\mathrm{H}$-bonds & 0.8 & 0.76 & 0.71 & 0.73 \\
\hline protein-water $\mathrm{H}$-bonds/total accessibility & 0.84 & 0.81 & 0.78 & 0.73 \\
\hline
\end{tabular}

The correlation coefficients are evaluated between the molecular weights and the different types of $\mathrm{H}$-bonds, the total solvent accessibility and its components, the number of water molecules and the related $\mathrm{H}$-bonds between residue proteins and water molecules, and void number and total void volumes (calculated with probe $=0$ ) obtained for each protein belonging to "mainly-alpha, "mainly-beta", "alpha/beta" and "alpha+beta" classes. The last row reports the correlation coefficient between the total accessibility and the number of $\mathrm{H}$-bonds between residue proteins and water molecules. 
Table 3: Mean ratios between the components and related total values for some properties.

\begin{tabular}{lcccc}
\hline A & mainly-alpha & mainly-beta & alpha/beta & alpha+beta \\
\hline MM-type/total & $0.84( \pm 0.10)$ & $0.74( \pm 0.14)$ & $0.74( \pm 0.08)$ & $0.77( \pm 0.11)$ \\
MS-type/total & $0.04( \pm 0.03)$ & $0.09( \pm 0.07)$ & $0.08( \pm 0.03)$ & $0.08( \pm 0.04)$ \\
SM-type/total & $0.07( \pm 0.05)$ & $0.10( \pm 0.07)$ & $0.09( \pm 0.04)$ & $0.08( \pm 0.05)$ \\
SS-type/total & $0.05( \pm 0.05)$ & $0.07( \pm 0.07)$ & $0.09( \pm 0.04)$ & $0.07( \pm 0.05)$ \\
B & & & \\
non-polar/total & $0.59( \pm 0.06)$ & $0.57( \pm 0.05)$ & $0.57( \pm 0.03)$ & $0.56( \pm 0.04)$ \\
polar/total & $0.41( \pm 0.06)$ & $0.43( \pm 0.05)$ & $0.43( \pm 0.03)$ & $0.44( \pm 0.04)$ \\
C & & & \\
surface/total void volume & $(0.95 \pm 0.05)$ & $0.96( \pm 0.06)$ & $0.85( \pm 0.07)$ & $0.92( \pm 0.07)$ \\
buried/total void volume & $0.05( \pm 0.04)$ & $0.04( \pm 0.03)$ & $0.15( \pm 0.07)$ & $0.08( \pm 0.06)$ \\
surface/total void number & $0.91( \pm 0.08)$ & $0.93( \pm 0.07)$ & $0.76( \pm 0.08)$ & $0.86( \pm 0.08)$ \\
buried/total void number & $0.09( \pm 0.08)$ & $0.07( \pm 0.07)$ & $0.24( \pm 0.08)$ & $0.14( \pm 0.08)$
\end{tabular}

(A) H-bonds, (B) accessible surface area, (c) void. Standard deviations are reported in parentheses.

A

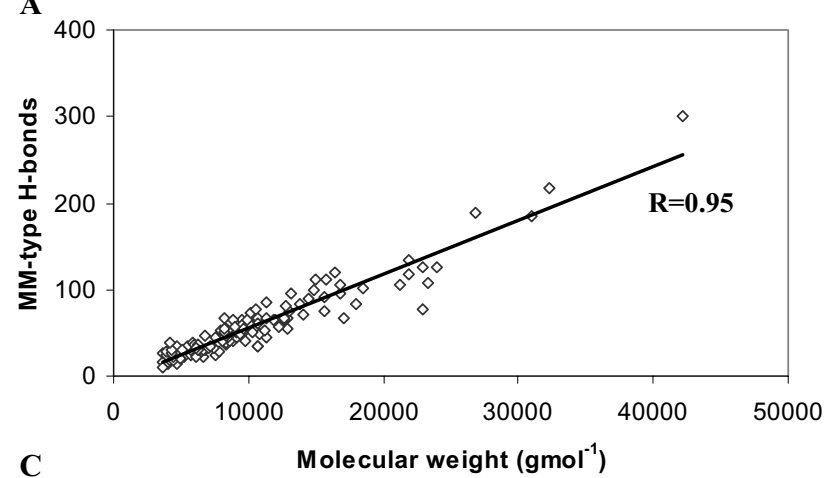

C

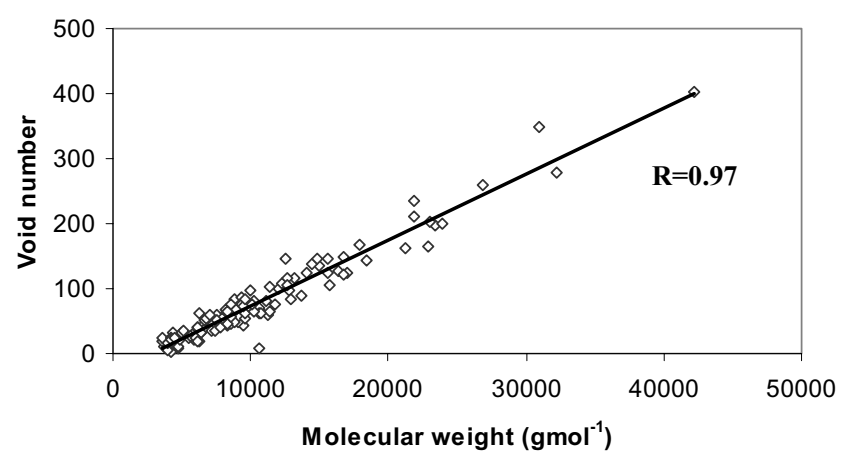

B
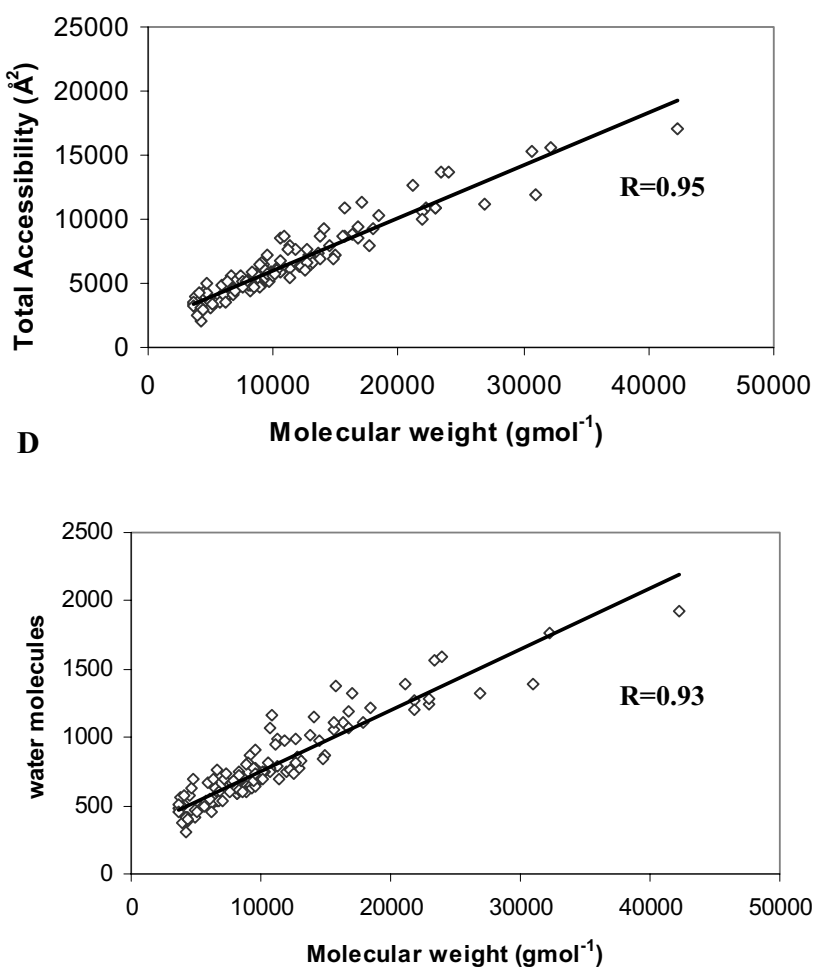

Figure I

Parameters plotted against values of molecular weights obtained for each protein belonging to "mainly-alpha" class. (A) MM-type H-bonds (B) total accessibility (C) void number (D) water molecules. The data were fit by linear least squares (the equations obtained are in Additional file 4). The related correlation coefficients (R) are also reported. 
beta" and "alpha+beta" proteins. The remaining $10 \%$ of analyzed structures presents long amino acid segments in irregular secondary structure at the beginning or end of the chain, which might explain their higher score value. These score values were then similarly computed to evaluate the structural quality of the theoretical protein models.

\section{Testing protein evaluation}

Targets from the CASP6 experiment were selected as a testing set, choosing only full-chain structures (see Methods for selection criteria). The list of the thirteen target models selected is reported in Table 4. These models were analyzed using the procedure reported above and their score values were calculated. Of the total number of models $54.6 \%$ (2285) showed scores higher than the threshold value, indicating that a large number of the theoretical models submitted to the CASP competition do not agree with the expected globularity features. It should also be noted that all those models considered to be the best submitted for each target $[16,17]$, as well as the relative experimental structures deposited in $\mathrm{PDB}$, had score values lower than the threshold value (Table 4). This confirms the quality of the best theoretical models submitted to the competition, and likewise confirms the reliability of our evaluation score.

\section{Discussion}

In this work four sets of protein structures were selected from the PDB, classified as "mainly-alpha", "mainlybeta", "alpha+beta" and "alpha/beta" (see Table 1) and analyzed in terms of H-bonds, void number, solventaccessible surface area and water molecules, comprised in a layer of 5 Ångstroms.
The mean ratios between MM-, MS-, SM- or SS-type Hbonds and the total number of $\mathrm{H}$-bonds, reported in Table 3A, indicated that the MM-type H-bonds were the most frequent in all four structural classes. This agreed with the previous analysis applied to a set of globular proteins, which had found that most $\mathrm{H}$-bonds were local and situated between backbone atoms in proteins, and that almost all were within single elements of the secondary structure [3]. The total number of MM-type H-bonds increased linearly with the molecular weight of selected proteins with correlation coefficients higher than 0.9 in "mainly-alpha", "alpha+beta" and "alpha/beta" proteins (Table 2, Figures 1 and Additional Files 1, 2, 3). In "mainly-beta" proteins the total number of $\mathrm{H}$-bonds was smaller than in other sets, with the correlation coefficient between the MM-type H-bonds and the protein molecular weights found to be 0.83 . This smaller value could depend on the higher content of irregular secondary structure in "mainly-beta" proteins (see Table 1).

The total accessible surface area (ASA), with its polar and non-polar components, was evaluated for all proteins of the four structural classes. The correlation coefficients between the non-polar components and the molecular weights were found to be higher than those obtained for the polar components in "mainly-alpha", "mainly-beta" and "alpha/beta" proteins, but not in "alpha+beta" proteins. In fact, in these structures the non-polar component was slightly higher than the polar one, as indicated by the mean ratios between non-polar accessibility values and the relative total accessibility (Table 3B).

Moreover, as for the $5 \AA$ layer around each protein, we verified that the number of water molecules increased line-

Table 4: Target proteins used as testing dataset.

\begin{tabular}{|c|c|c|c|c|c|}
\hline Target & Class & PDB code & Score value for PDB structures & Number of models & $\begin{array}{l}\text { Percentage of models } \\
\text { with score } \geq 5.9 \text { or } \geq 5.1\end{array}$ \\
\hline T0198 & mainly-alpha & ISUM_3-223 & 5.1 & 154 & 90.9 \\
\hline T0238 & mainly-alpha & IW33_70-222 & 5.8 & 154 & 86.4 \\
\hline T0209_I & mainly-beta & IXQB_9-I38 & 3.11 & 152 & 87.6 \\
\hline T0212 & mainly-beta & ITZA_3-I2I & 3.4 & 182 & 41.2 \\
\hline T0199_3 & alpha+beta & ISTZ_|45-226 & 2.10 & 223 & 50.7 \\
\hline T020I & alpha+beta & ISI2_I-90 & 1.6 & 204 & 20.6 \\
\hline T0209_2 & alpha+beta & IXQB_I59-23I & 2.35 & 229 & $4 I$ \\
\hline T02 I6_I & alpha+beta & IVL4_2-2I4 & 4.3 & 155 & 93.5 \\
\hline T0216_2 & alpha+beta & IVL4_22I-433 & 3.3 & 147 & 95.3 \\
\hline T0239 & alpha+beta & IRKI_I-98 & 1.8 & 251 & 29.1 \\
\hline T0248_2 & alpha+beta & ITD6_107-193 & 2.9 & 239 & 32.6 \\
\hline T0242 & alpha/beta & 2BLK_2-II6 & 3.1 & 200 & 43 \\
\hline T0273 & alpha/beta & IWDJ_2-187 & 1.2 & 141 & 90 \\
\hline
\end{tabular}

The columns report the target codes, structural class, code of the experimental structure deposited in the PDB with the segment analyzed, their score value, the number of full-atom models analyzed in this work, and the percentage of models for which the score value resulted over the threshold (i.e. $\geq 5.9$ for "mainly-alpha" and "mainly-beta" proteins and $\geq 5.1$ for "alpha/beta" and "alpha+beta" proteins). 
arly with the molecular weight of the proteins in all four sets (Figures 1 and Additional Files 1, 2, 3). The correlation coefficients, calculated for proteins belonging to all four classes, between water-protein H-bonds and the relative total accessibility values were found to be consistently higher than those between water-protein $\mathrm{H}$-bonds and the protein molecular weights (Table 2). This suggests that both protein shape and surface extension may have a greater effect on the number of $\mathrm{H}$-bond interactions between proteins and water than does protein size.

Finally, the voids were analyzed in all proteins to assess both packing quality and individual voids $[18,19]$. The correlation coefficient between the largest void volumes and the total void volumes, calculated for all proteins belonging to each given class, was 0.76 in "mainly-alpha", 0.71 in "mainly-beta", 0.71 in "alpha+beta" and 0.58 in "alpha/beta" proteins, respectively. These values indicated that the total void volume of each structure belonging to "mainly-alpha", "mainly-beta" and "alpha+beta" classes represents mainly the largest void present, in agreement with results obtained analyzing a heterogeneous dataset [18]. The mean ratios between the total surface and buried void volumes and the total void volume (Table $3 \mathrm{C}$ ) indicated that the selected structures had a greater number of voids on the surface and few buried voids, and that the total void volume consisted mainly of the surface void volume. The presence of few buried voids was used to verify that the selected proteins are well-packed and compact $[7,20]$. Moreover, the total void volumes are $<1500 \AA^{3}$ in "mainly-alpha" and "mainly-beta" proteins, and $>3000 \AA$ 3 in those "alpha/beta" and "alpha+beta", in which more buried voids are also present (see Table 3C). These values could indicate that the "mainly-alpha" and "mainly-beta" proteins are more compact than the others.

As a final comment on the evaluation of these globularity features, we note that some differences were seen among the four structural classes, whose cause requires further investigation. Different degrees of compactness as well as flexibility may be needed depending on the structural architecture which is often related to the functional role. As an example, the multi domain nature of alpha+beta proteins is often related to the need to open the protein globe and offer a larger cavity to fit a ligand. Likewise, as in the case of barrel architecture, alpha/beta proteins show a large internal cavity which could explain the differences in void ratios for this class. However, other hypotheses could be postulated and this aspect of the results warrants further investigation.

Our analyses have shown that some globular properties are well conserved in proteins within the same structural class. Therefore, from these studies we obtained some theoretical parameters, i.e. linear regression equations and
Root Mean Squared Error (RMSE), shown in Additional File 4, specific for each structural class. These parameters could be useful in evaluating models, especially those predicted by "ab-initio" methods, for which reference structures are not available. A score value for all proteins was calculated by using the parameters having the highest correlation coefficients with the protein molecular weights (MM-type H-bonds, void number, total accessible surface area and water molecules) (see Figure 2).

To test the applicability and usefulness of these criteria, thirteen targets of CASP experiment were selected. Only the full-atom structures were chosen for each testing set $[16,17]$. Our results surprisingly show that many of the models submitted (54.6\%) should be discarded a priori because they do not have the structural properties expected in globular proteins.

An interesting aspect concerns the subdivision of prediction methods present in CASP6 as "human" and "server" predictors [21]. The results shown in Table 5 separate the two classes of predictors. The models exceeding the threshold value of our globularity score were $51.6 \%$ and $64.2 \%$ for human and server predictors, respectively.

The threshold value has been applied in our analysis as a cutoff which creates two subsets of models, the one below the cutoff should include models with globularity features in agreement with those expected in crystallographic structures, while the subset of models above the cutoff should include models with poor globularity features. To validate this, we evaluated the average model quality for the two subsets by using root-mean square deviations (RMSD) and Global Distance Test Total Score (GDT_TS) reported in CASP6 tables, as well as MaxSub score [22], by considering these parameters as correct model quality measures.

For each target and for the whole set, the average values of RMSD and GDT_TS were evaluated for the two subsets of models (see Additional File 5). The subset of the models below the cutoff resulted always (i.e., in the whole set as well as for each target) to have the best average quality, compared to the other subset.

Moreover, we correlated our globularity score with a number of model evaluation parameters, i.e. gross violations of distance constraints (err), RMSD and GDT_TS reported in CASP6 tables, as well as MaxSub score. In addition, we applied to the models other quality assessment programs, i.e. ProsaII Z-score [23], Modcheck score [24], Anolea Z-score [25,26], and Victor/FRST function [27], and compared these scores with our globularity score. The plots for each comparison are shown in Additional Files $6,7,8,9,10,11,12,13,14,15,16,17,18,19$, 
A

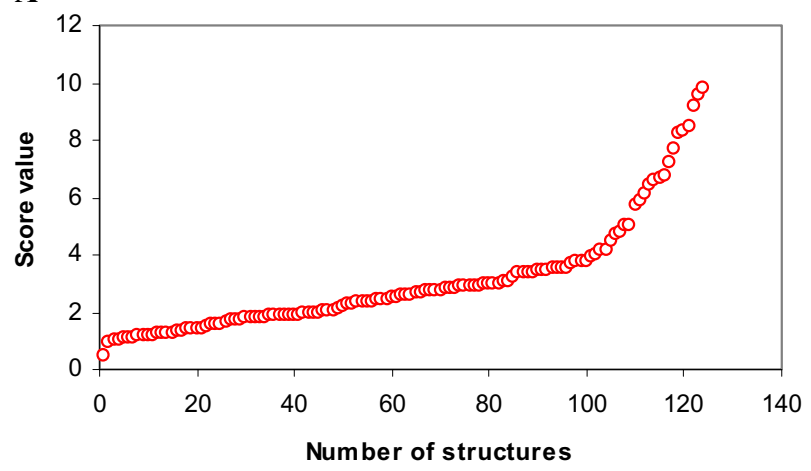

C

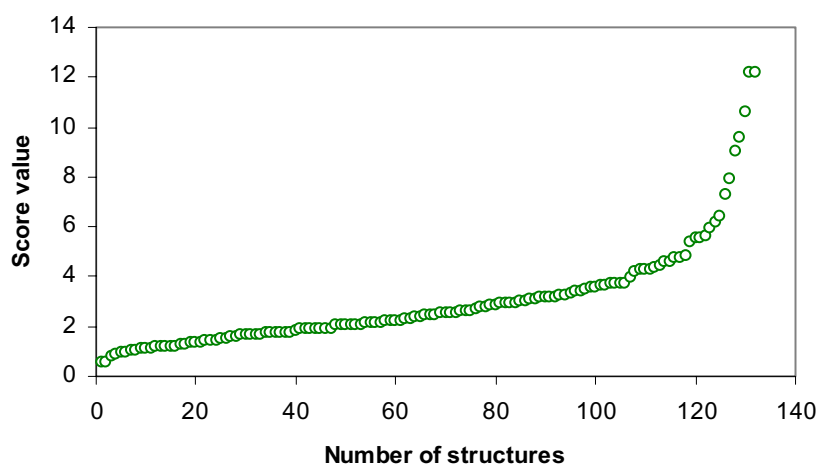

B

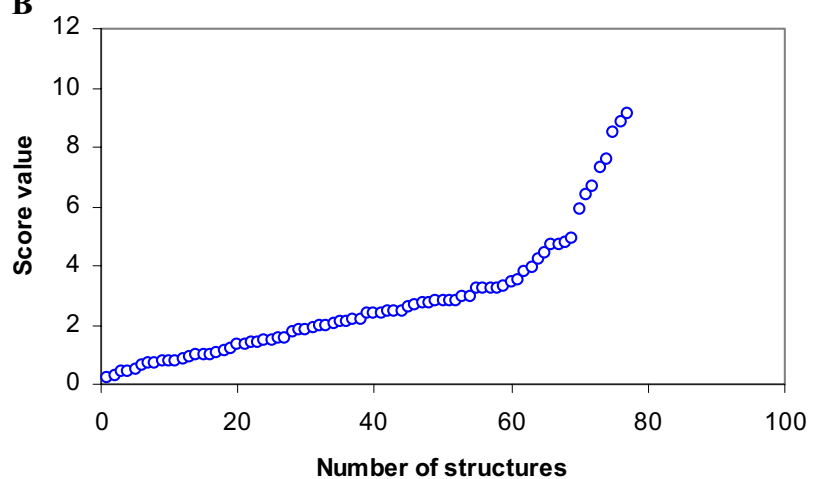

D

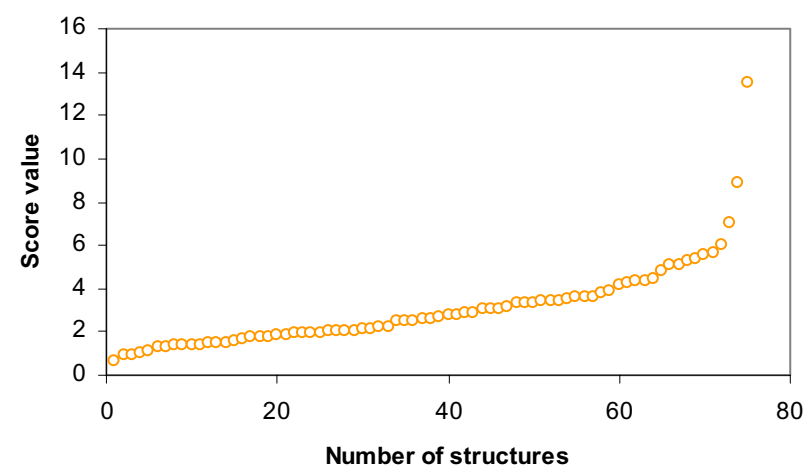

\section{Figure 2}

Score value calculated for all the proteins belonging to the four structural classes. (A) "mainly-alpha", (B) "mainlybeta", (C) "alpha+beta" and (D) "alpha/beta". The score values are calculated summing for all the proteins the ratios of the differences between the calculated and predicted values for each of four properties (number of MM-type H-bonds, void and water molecules, and total accessibility) versus the related RMSE.

corresponding to each of thirteen targets analyzed and to the whole set. In particular, $79 \%$ of models with globularity scores lower than the threshold values have less than 3 distance violation (err). Overall, the trend indicates that the threshold value of the globularity score selects the better models as evaluated by the different parameters. The linear correlation coefficients suggest that the globularity score is not directly related to ProsaII Z-score, Modcheck score, Anolea Z-score, and Victor/FRST function. This suggests that our globularity score cannot simply be derived from other evaluation parameters, which means that our score can be used as an independent evaluation criterion.

The correlation coefficients between our globularity score, as well as all quality assessment programs listed above, and the correct quality measures (i.e. RMSD, GDT_TS and MaxSub) were evaluated for the whole set (see Table 6) and for thirteen targets (see Additional File 20). The globularity score has the better correlation with RMSD and GDT_TS.
In Tables 6 and Additional File 20 we have also reported the correlation coefficients between the four individual features (MM-type H-bonds, void number, water molecules and total accessibility) used in our work and the correct measures (RMSD, GDT_TS and MaxSub).

The combination of the four parameters in our score offer certainly some advantage. In fact, we have also evaluated the number of models in every dataset having the void number, MM-type H-bonds, water molecules and total accessibility in the ranges defined by the predicted values \pm RMSE (see Additional Files 4 and 21-A). These results showed that the four structural properties are independent. The average GDT_TS measures for the models which pass the selection with the single features were also evaluated in order to know their quality (see Additional File 21B). The average GDT_TS values for the whole set of selected models range from 22.4 to 22.9. By considering that the average GDT_TS for the subsets below and above the cutoff (see Additional File 5) were 24.1 and 15.2, 
Table 5: Details for the analysis of testing datasets.

\begin{tabular}{|c|c|c|c|c|c|c|}
\hline & \multicolumn{3}{|c|}{ Total number Models } & \multicolumn{3}{|c|}{ Model within expected score } \\
\hline & Total & Human & Server & Total & Human & Server \\
\hline T0198 & 152 & $110 / 152$ & $42 / 152$ & $14 / 152$ & $9 / 110$ & $5 / 42$ \\
\hline T0238 & 154 & $117 / 154$ & $37 / 154$ & $21 / 154$ & $18 / 117$ & $3 / 37$ \\
\hline T0209_I & 152 & $108 / 152$ & $43 / 152$ & $20 / 152$ & $20 / 108$ & $0 / 43$ \\
\hline $\mathrm{T0212}$ & $|8|$ & |35/|8| & $46 / 181$ & $107 / 181$ & $87 / 135$ & $20 / 46$ \\
\hline T0199_3 & 223 & $177 / 223$ & $46 / 223$ & $110 / 223$ & $96 / 177$ & $14 / 46$ \\
\hline T020I & 203 & $165 / 203$ & $38 / 203$ & $162 / 203$ & $136 / 165$ & $26 / 38$ \\
\hline T0209_2 & 229 & $172 / 229$ & $57 / 229$ & $135 / 229$ & $103 / 172$ & $32 / 57$ \\
\hline T0216_I & 155 & $123 / 155$ & $32 / 155$ & $10 / 155$ & $6 / 123$ & $4 / 32$ \\
\hline T0216_2 & 147 & I |4/147 & $33 / 147$ & $7 / 147$ & $6 / 114$ & $1 / 33$ \\
\hline T0239 & 251 & $207 / 251$ & $44 / 251$ & $|78 / 25|$ & $|50 / 25|$ & $28 / 44$ \\
\hline T0248_2 & 238 & $180 / 238$ & $58 / 238$ & $161 / 238$ & $125 / 180$ & $36 / 58$ \\
\hline T0242 & 200 & $154 / 200$ & $46 / 200$ & $1 \mid 4 / 200$ & $96 / 154$ & $18 / 46$ \\
\hline T0273 & 141 & $107 /|4|$ & $34 / 141$ & $|4 /| 4 \mid$ & $10 / 107$ & $4 / 34$ \\
\hline
\end{tabular}

For each target, columns report the total number of models analyzed, the ratio of models from "human" and "server" predictors, the ratios of models which have globularity score below the threshold value.

respectively, we observe that the subset with the better quality is that selected by the globularity score. The exclusion of models by considering the simple range of each parameter, or the combination of two, three, or four parameters (see Additional File 22) may be too restrictive and in some cases would even disqualify the best theoretical models submitted for the targets. On the contrary, the use of the globularity score and the related value allows some models to pass the threshold even when some of four parameters do not fall within the range.

Moreover, all the experimental structures, deposited in $\mathrm{PDB}$, and the theoretical models indicated as the best submitted for these targets $[16,17]$, had score values below the thresholds ( $\leq 5.1$ or $\leq 5.9$, depending on the structural class). This confirms that our method provides reliable results. It should be noted that globularity is a spread property of proteins, but some proteins may have poor globularity features. Therefore, we strongly suggest using our scoring method only for proteins expected to be globular. Finally, our study suggests that the evaluation of theoretical models can be improved by taking into account the globularity features before releasing the models, submitting them to CASP, or using them for further studies.

\section{Conclusion}

We have analyzed structural properties that characterize protein globularity and have suggested an operative procedure to be used for the analysis of globular quality of theoretical protein models, obtained by computational approaches in the absence of experimental target structures. Our scoring method is a tool to avoid the diffusion of incorrect structures and of incorrect functional hypotheses, that can be employed to check the globularity of predicted models and to supplement other methods already used to evaluate their quality $[28,29]$.

Table 6: Correlation coefficients for whole set of models.

\begin{tabular}{lccc}
\hline & RMS & GDT_TS & MaxSub \\
\hline Z-score [Prosa] & 0.16 & 0.04 & -0.026 \\
Modcheck score & -0.18 & -0.11 & -0.05 \\
Victor/FRST function & 0.16 & -0.35 & -0.27 \\
Z-score [Anolea] & 0.16 & -0.04 & -0.14 \\
Globularity score & 0.56 & -0.4 & -0.074 \\
MM-type H-bonds & 0.25 & -0.12 & 0.37 \\
Void number & -0.17 & 0.036 & -0.093 \\
Total accessibility & 0.51 & -0.40 & 0.0066 \\
Water molecules & 0.54 & -0.36 & 0.077 \\
\hline
\end{tabular}

In columns we report the correlation coefficients between the quality assessment methods (Prosa, Modcheck, Victor/FRST, Anolea and Globularity score), as well as our four individual features (MM-type H-bonds, void number, water molecules and total accessibility), and three correct quality measures (i.e. RMSD, GDT_TS and MaxSub) 


\section{Methods}

\section{Training datasets and their analysis}

The list of protein structures used in this study was obtained using PDBselect, a set of experimentally determined, non-redundant protein structures in the PDB. The secondary structure for each PDB entry was assigned by the DSSP algorithm based on the analysis of backbone dihedral angles and hydrogen bonds. DSSP assigns seven different secondary structures, i.e., H: alpha-helix, G: $3_{10}$ helix, I: $\pi$-helix, E: extended strand, B: residue in isolated beta-bridge, S: bend, and T: H-bonded turn. In addition, a "coil" state is assigned when no secondary structure is recognized [13]. We applied the convention to define H, G, I as helix, E and B as strand, and others as coil [30].

A Perl script was written to select the monomeric protein structures, that contain only alpha-helices ("mainlyalpha"), only beta- strands ("mainly-beta") and alphahelices and beta- strands ("alpha-beta") on the basis of the clearly defined classification [31]. The "alpha-beta" proteins were subdivided in "alpha+beta" and "alpha/ beta" on the basis of SCOP classification [14,15]. For the structures determined by NMR, only one chain in the file was considered.

Hydrogen atoms were added to the protein structures with the "Modify/Add Hydrogens tool" in InsightII package (Accelrys, Inc., San Diego, CA). The Hbplus package was used to evaluate the putative formation of H-bonds. It identifies H-bonds within a distance of 2.5 Ångstroms and a minimum angle of $90^{\circ}$ [32]. Solvent accessibility of amino acids was evaluated by the NACCESS program, calculating the atomic accessible surface defined by rolling a probe of 1.40 Ångstroms around the van der Waals surface of every protein structure [33]. The AVP program was used to analyze voids in proteins, defined as empty cavities not accessible to solvent [18]. It uses a simple grid-based method and separates the probe size used to define voids from the probe size used to define channels to the surface. This method combines the analysis of individual discrete voids with that of packing quality and can be applied to the calculation of total void volume, maximum void size, and number of voids.

A layer of water molecules around every protein was added using the tool "Assembly $\rightarrow$ Soak $\rightarrow$ Layer" in InsightII. The H-bonds between the residue atoms in protein and the water molecules was evaluated by Hbplus program.

Further Perl scripts were written to apply the AVP, Hbplus and NACCESS programs to the selected protein dataset.

\section{Linear regression and $R M S E$}

The total accessible surface area (ASA), the number of MM-type H-bonds, voids and water molecules versus the molecular weights of selected proteins in each structural class fit to linear regressions described by four equations relating the $y$ values (i.e. $y_{A S A^{\prime}} y_{M M^{\prime}} y_{\text {void }}$ and $y_{\text {water }}$ ) to the $x$ values (i.e. molecular weights). For each of four properties the Root Mean Squared Error (RMSE) was calculated, that is the average distance of all the points from the fitted line, measured along a vertical line

$R M S E=E_{i}^{C}=\sqrt{\frac{1}{N^{C}} \sum_{i=1}^{N}\left(y_{i}-y_{i}^{\prime}\right)^{2}}$

where $N_{C}$ is the total number of structures in each class, $y_{i}$ is the value predicted by linear equation in each of the four cases (i.e. $y_{A S A}, y_{M M}, y_{\text {void }}$ and $y_{\text {water }}$ ) and $y_{i}^{\prime}$ is the corresponding calculated value.

\section{Score value}

A score value was calculated for all the proteins, belonging to one class, by summing the ratios of the differences between the calculated and predicted values for each of the four properties versus the related errors $\left(E_{i}{ }^{C}\right)$.

$\sigma^{C}=\left[\frac{\left|y_{A S A}-y_{A S A}^{\prime}\right|}{E_{A S A}^{C}}+\frac{\left|y_{M M}-y_{M M}^{\prime}\right|}{E_{M M}^{C}}+\frac{\left|y_{\text {void }}-y_{\text {void }}^{\prime}\right|}{E_{\text {void }}^{C}}+\frac{\left|y_{\text {water }}-y_{\text {water }}^{\prime}\right|}{E_{\text {water }}^{C}}\right]$

As for the most frequent score values, calculated for the proteins belonging to the same class, it was possible to identify a score value specific for each of the four structural classes. These score values were then used to evaluate the structural properties of models predicted by folding "ab-initio" methods and used as "testing dataset".

\section{Testing protein dataset}

The testing structures were selected starting from all the protein models predicted for the CASP6 protein structure prediction experiment in the New Fold (NF) and difficult Fold Recognition Analogous (FR/A) categories [16,17]. To analyze globularity features, we selected only those models for which complete chains are available, for both the experimental and the theoretical structures. Following these criteria thirteen targets were selected, i.e. T0198 (PDB code: 1SUM (3-223)) and T0238 (1W33 (70-222)) classified as "mainly-alpha", T0212 (1TZA (3-121)) and T0209_1 (1XQB (9-138)) as "mainly-beta", T0199_3 (1STZ (145-226)), T0201 (1S12 (1-90)), T0209_2 (1XQB(159-221), T0216_1 (1VL4(2-214)), T0216_2 (1VL4(221-433)), T0239 (1RKI(1-98)) and T0248_2 (1TD6 (107-193)) as "alpha+beta" and T0242 (2BLK (2116)) and T0273 (1WDJ(2-187)) as "alpha/beta". All theoretical models submitted for each of these targets 
were used as testing structures, but only if the full atom model was available. These models were analyzed with the same software used for the training protein structures and their structural properties were evaluated by the score values.

Next, our globularity scores were compared with some model evaluation parameters, i. e. gross violations of distance constraints (err), root-mean square deviations (RMSD) and Global Distance Test_Total Score (GDT_TS) reported in CASP6 tables.

Finally, all models were analyzed also by MaxSub program [22], PROSA II Z-score [23], Modcheck score [24], Anolea Z-score [25,26], and Victor/FRST function [27], using the default parameters for each of these. Briefly, MaxSub measures the similarity of a model to its corresponding experimental structure reporting 0 for a completely wrong model and 1 for a perfect model. Prosa II and Anolea compute a Z-score value indicating the better model on the basis of the lower value but Modcheck on the basis of the higher value. Victor/FRST package calculates a pseudo-energy to evaluate the quality of a given protein structural model for which the lower score reflects the better model.

\section{Web site}

A web site has been devoted to the additional materials we produced during this work and to add in the time more evaluations. The site is freely accessible [34].

\section{Authors' contributions}

AMF and GC conceived of the study and participated in its design and coordination. SC carried out the computational experiments and analysis of the results. All authors read and approved the final manuscript.

\section{Additional material}

\section{Additional File 1}

Figure1S. Parameters plotted against values of molecular weights obtained for each protein, belonging to "mainly-beta" class. (A) MM-type H-bonds (B) total accessibility (C) void number (D) water molecules. Click here for file

[http://www.biomedcentral.com/content/supplementary/14726807-7-9-S1.pdf]

\section{Additional File 2}

Figure2S. Some parameters plotted against values of molecular weights obtained for each protein, belonging to "alpha/beta" class. (A) MM-type $H$-bonds (B) total accessibility (C) void number (D) water molecules. Click here for file

[http://www.biomedcentral.com/content/supplementary/14726807-7-9-S2.pdf]

\section{Additional File 3}

Figure3S. Some parameters plotted against values of molecular weights obtained for each protein, belonging to "alpha+beta" class. (A) MM-type $H$-bonds (B) total accessibility (C) void number (D) water molecules. Click here for file

[http://www.biomedcentral.com/content/supplementary/14726807-7-9-S3.pdf]

\section{Additional File 4}

Table1S. Theoretical parameters obtained analysing protein structures belonging to four structural classes.

Click here for file

[http://www.biomedcentral.com/content/supplementary/14726807-7-9-S4.pdf]

\section{Additional File 5}

Table2S. We have reported for each target and for the whole model set the cutoff value of globularity score, and the average values of RMSD and GDT_TS for the models above and below the cutoff. In parenthesis are reported the standard deviations.

Click here for file

[http://www.biomedcentral.com/content/supplementary/14726807-7-9-S5.pdf]

\section{Additional File 6}

Figure4S. Model evaluation parameters plotted against values of globularity score obtained for T0198 target (A) gross violations of distance constraints (err) (B) root-mean square deviations (RMSD) (C) Global Distance Test_Total Score (GDT_TS) (D) MaxSub score (E) PROSA II $Z$-score (F) Victor/FRST function (G) Modcheck score (H) Anolea Zscore.

Click here for file

[http://www.biomedcentral.com/content/supplementary/14726807-7-9-S6.pdf]

\section{Additional File 7}

Figure5S. Model evaluation parameters plotted against values of globularity score obtained for T0201 target (A) gross violations of distance constraints (err) (B) root-mean square deviations (RMSD) (C) Global Distance Test_Total Score (GDT_TS) (D) MaxSub score (E) PROSA II $Z$-score (F) Victor/FRST function (G) Modcheck score (H) Anolea Zscore.

Click here for file

[http://www.biomedcentral.com/content/supplementary/14726807-7-9-S7.pdf]

\section{Additional File 8}

Figure6S. Model evaluation parameters plotted against values of globularity score obtained for T0212 target (A) gross violations of distance constraints (err) (B) root-mean square deviations (RMSD) (C) Global Distance Test_Total Score (GDT_TS) (D) MaxSub score (E) PROSA II $Z$-score (F) Victor/FRST function (G) Modcheck score (H) Anolea Zscore.

Click here for file

[http://www.biomedcentral.com/content/supplementary/14726807-7-9-S8.pdf] 


\section{Additional File 9}

Figure7S. Model evaluation parameters plotted against values of globularity score obtained for T0238 target (A) gross violations of distance constraints (err) (B) root-mean square deviations (RMSD) (C) Global Distance Test_Total Score (GDT_TS) (D) MaxSub score (E) PROSA II $Z$-score (F) Victor/FRST function (G) Modcheck score (H) Anolea Zscore.

Click here for file

[http://www.biomedcentral.com/content/supplementary/14726807-7-9-S9.pdf]

\section{Additional File 10}

Figure8S. Model evaluation parameters plotted against values of globularity score obtained for T0242 target (A) gross violations of distance constraints (err) (B) root-mean square deviations (RMSD) (C) Global Distance Test_Total Score (GDT_TS) (D) MaxSub score (E) PROSA II Z-score (F) Victor/FRST function (G) Modcheck score (H) Anolea Zscore.

Click here for file

[http://www.biomedcentral.com/content/supplementary/14726807-7-9-S10.pdf]

\section{Additional File 11}

Figure9S. Model evaluation parameters plotted against values of globularity score obtained for T0248 target (A) gross violations of distance constraints (err) (B) root-mean square deviations (RMSD) (C) Global Distance Test_Total Score (GDT_TS) (D) MaxSub score (E) PROSA II Z-score (F) Victor/FRST function (G) Modcheck score (H) Anolea Zscore.

Click here for file

[http://www.biomedcentral.com/content/supplementary/14726807-7-9-S11.pdf]

\section{Additional File 12}

Figure10S. Model evaluation parameters plotted against values of globularity score obtained for T0216_1 target (A) gross violations of distance constraints (err) (B) root-mean square deviations (RMSD) (C) Global Distance Test_Total Score (GDT_TS) (D) MaxSub score (E) PROSA II $Z$-score (F) Victor/FRST function (G) Modcheck score (H) Anolea Zscore.

Click here for file

[http://www.biomedcentral.com/content/supplementary/14726807-7-9-S12.pdf]

\section{Additional File 13}

Figure11S. Model evaluation parameters plotted against values of globularity score obtained for T0216_2 target (A) gross violations of distance constraints (err) (B) root-mean square deviations (RMSD) (C) Global Distance Test_Total Score (GDT_TS) (D) MaxSub score (E) PROSA II $Z$-score (F) Victor/FRST function (G) Modcheck score (H) Anolea Zscore.

Click here for file

[http://www.biomedcentral.com/content/supplementary/14726807-7-9-S13.pdf]

\section{Additional File 14}

Figure12S. Model evaluation parameters plotted against values of globularity score obtained for T0239 target (A) gross violations of distance constraints (err) (B) root-mean square deviations (RMSD) (C) Global Distance Test_Total Score (GDT_TS) (D) MaxSub score (E) PROSA II Z-score (F) Victor/FRST function (G) Modcheck score (H) Anolea Zscore.

Click here for file

[http://www.biomedcentral.com/content/supplementary/14726807-7-9-S14.pdf]

\section{Additional File 15}

Figure13S. Model evaluation parameters plotted against values of globularity score obtained for T0199_3 target (A) gross violations of distance constraints (err) (B) root-mean square deviations (RMSD) (C) Global Distance Test_Total Score (GDT_TS) (D) MaxSub score (E) PROSA II $Z$-score (F) Victor/FRST function (G) Modcheck score (H) Anolea Zscore.

Click here for file

[http://www.biomedcentral.com/content/supplementary/14726807-7-9-S15.pdf]

\section{Additional File 16}

Figure14S. Model evaluation parameters plotted against values of globularity score obtained for T0209_1 target (A) gross violations of distance constraints (err) (B) root-mean square deviations (RMSD) (C) Global Distance Test_Total Score (GDT_TS) (D) MaxSub score (E) PROSA II $Z$-score (F) Victor/FRST function (G) Modcheck score (H) Anolea Zscore.

Click here for file

[http://www.biomedcentral.com/content/supplementary/14726807-7-9-S16.pdf]

\section{Additional File 17}

Figure15S. Model evaluation parameters plotted against values of globularity score obtained for T0209_2 target (A) gross violations of distance constraints (err) (B) root-mean square deviations (RMSD) (C) Global Distance Test_Total Score (GDT_TS) (D) MaxSub score (E) PROSA II $Z$-score (F) Victor/FRST function (G) Modcheck score (H) Anolea Zscore.

Click here for file

[http://www.biomedcentral.com/content/supplementary/14726807-7-9-S17.pdf]

\section{Additional File 18}

Figure16S. Model evaluation parameters plotted against values of globularity score obtained for T0273 target (A) gross violations of distance constraints (err) (B) root-mean square deviations (RMSD) (C) Global Distance Test_Total Score (GDT_TS) (D) MaxSub score (E) PROSA II $Z$-score (F) Victor/FRST function (G) Modcheck score (H) Anolea Zscore.

Click here for file

[http://www.biomedcentral.com/content/supplementary/14726807-7-9-S18.pdf]

\section{Additional File 19}

Figure17S. Model evaluation parameters plotted against values of globularity score obtained for all models (A) gross violations of distance constraints (err) (B) root-mean square deviations (RMSD) (C) Global Distance Test_Total Score (GDT_TS) (D) MaxSub score (E) PROSA II $Z$-score (F) Victor/FRST function (G) Modcheck score (H) Anolea Zscore.

Click here for file

[http://www.biomedcentral.com/content/supplementary/14726807-7-9-S19.pdf]

\section{Additional File 20}

Table3S. Correlation coefficients between all methods, as well as for our four individual features (MM-type H-bonds, void number, water molecules and total accessibility), and three correct quality measures (i.e. RMSD, GDT_TS and MaxSub) evaluated for each of 13 target. Click here for file [http://www.biomedcentral.com/content/supplementary/14726807-7-9-S20.pdf] 


\section{Additional File 21}

Table4S. A. For each target, columns report the number of models analyzed and the ratios of models for which the voids, MM-type H-bonds, water molecules, the total accessibility and the score values resulted within expected ranges for globular proteins and calculated as reported in Methods (see Additional File 4). B. For each target and for the whole set, columns report average GDT_TS value evaluated for models that have the number of void, MM-type H-bonds and water molecules, and the total accessibility within the expected range. In parenthesis are reported the standard deviations.

Click here for file

[http://www.biomedcentral.com/content/supplementary/14726807-7-9-S21.pdf]

\section{Additional File 22}

Table5S. Number of models for which the combination of two, three or four parameters (voids, MM-type H-bonds, water molecules and total accessibility) resulted in the expected ranges for globular proteins and calculated as reported in Methods (see Additional File 4).

Click here for file

[http://www.biomedcentral.com/content/supplementary/14726807-7-9-S22.pdf]

\section{Acknowledgements}

We thank Mrs. Tricia Reynolds for the careful revision of the manuscript. This work was partially supported by F.I.R.B. grant RBNE0I57EH_003, CNR-Bioinformatics Project, Progetto Oncoproteomica Conv. n.527B/2A/ 10.

\section{References}

I. Grigoryan G, Zhou F, Lusting SR, Ceder G, Morgan D, Keating AE: Ultra-fast evaluation of protein energies directly from sequence. PLOS Computational Biology 2006, 2:55I-563.

2. Pace CN, Trevino S, Prabhakaran E, Scholtz JM: Protein structure, stability and solubility in water and other solvents. Phil Trans $R$ Soc Lond B 2004, 359: I225-1235.

3. Stickle DF, Presta LG, Dill KA, Rose GD: Hydrogen bonding in globular proteins. J Mol Biol 1992, 226: I 143-1 I59.

4. Pauling L, Corey RB, Branson HR: The structure of proteins: two hydrogen-bonded helical configurations of the polypeptide chains. Proc Nat Acad Sci 195I, 37:205-2II.

5. Perutz MF: New X-ray evidence on the configuration of polypeptide chains. Nature 1951, 167:1053-1054.

6. Fersht AR: The hydrogen bond in molecular recognition. Trends Biochem Sci 1987, 1 2:301-304.

7. Hubbard SJ, Argos P: Evidence on close packing and cavities in proteins. Current Opinion in Biotechnology 1995, 6:375-38I.

8. Hubbard SJ, Gross K-H, Argos P: Intramolecular cavities in globular proteins. Protein Eng 1994, 7:6/3-626.

9. Fleming PJ, Richards FM: Protein Packing: Dependence on protein size, secondary structure and amino acid composition. J Mol Biol 2000, 299:487-498.

10. Linding R, Russell RB, Neduva V, Gibson TJ: GlobPlot: exploring protein sequences for globularity and disorder. Nucleic Acids Research 2003, 31:3701-3708.

1I. Rost B, Yachdav G, Liu J: The PredictProtein server. Nucleic Acids Research 2004, 32:W32I-W326.

12. Hobohm U, Scharf M, Schneider R, Sander C: Selection of a representative set of structures from the Brookhaven Protein Data Bank Protein. Protein Science 1992, 1:409-417.

13. Kabsch W, Sander C: Dictionary of protein secondary structure: pattern recognition of hydrogen-bonded and geometrical features. Biopolymers 1983, 22:2577-2637.

14. Murzin AG, Brenner SE, Hubbard T, Chothia C: SCOP: a structural classification of proteins database for the investigation of sequences and structures. J Mol Biol 1995, 247:536-540.
15. Andreeva A, Howorth D, Brenner SE, Hubbard T], Chothia C, Murzin AG: SCOP database in 2004: refinements integrate structure and sequence family data. Nucleic Acids Res 2004, 32:D226-D229.

16. Tress M, Tai C-H, Wang G, Ezkurdia I, Lopez G, Valencia A, Lee B-K, Dunbrack RL Jr: Domain definition and target classification for CASP6. Proteins 2005:8-18.

17. Vincent JJ, Tai C-H, Sathyanarayana BK, Lee B: Assessment of CASP 6 predictions for new and nearly new fold targets. Proteins 2005:67-83.

18. Cuff AL, Martin ACR: Analysis of void volumes in proteins and application to stability of the p53 tumour suppressor protein. J Mol Biol 2004, 344: I I99-I 209.

19. Tsai J, Taylor R, Chothia C, Gerstein M: The packing density in proteins: standard radii and volumes. J Mol Biol 1999 , 290:253-266.

20. Liang J, Edelsbrunner H, Fu P, Sudhakar PV, Subramaniam S: Analytical shape computation of macromolecules: II. Inaccessible cavities in proteins. Proteins 1998, 33:18-29.

21. Moult J, Fidelis K, Rost B, Hubbard T, Tramontano A: Critical assessment of methods of protein structure prediction (CASP) - round 6. Proteins 2005:3-7.

22. Siew N, Elofsson A, Rychlewski L, Fischer D: MaxSub: an automated measure for the assessment of protein structure prediction quality. Bioinformatics 200 I, 16:776-785.

23. Sippl MJ: Recognition of errors in three-dimensional structures of proteins. Proteins 1993, 17:355-362.

24. Pettitt CS, McGuffin LJ, Jones DT: Improving sequence-based fold recognition by using 3D model quality assessment. Bioinformatics 2005, 21:3509-35। 5 .

25. Melo F, Feytmans E: Novel knowledge-based mean force potential at atomic level. J Mol Biol 1997, 267:207-222

26. Melo F, Feytmans E: Assessment of protein structures based on the non-local energy. J Mol Biol 1998, 277: I I4I-II52.

27. Tosatto SC: The victor/FRST function for model quality estimation. J Comput Biol 2005, I 2:1316-1327.

28. Laskowski RA, MacArthur MW, Moss DS, Thornton JM: PROCHECK - A program to check the stereochemical quality of protein structures. J Appl Cryst 1993, 26:283-29I.

29. Sims GE, Kim S-H: A method for evaluating the structural quality of protein models by using higher-order phi-psi pairs scoring. PNAS 2006, 103:4428-4432.

30. Rost $B$, Eyrich VA: EVA: large-scale analysis of secondary structure prediction. Proteins 2001:192-199.

31. Levitt M, Chothia C: Structural pattern in globular proteins. Nature 1976, 261:552-557.

32. McDonald IK, Thornton JM: Satisfying hydrogen bonding potential in proteins. J Mol Biol 1994, 238:777-793.

33. Hubbard SJ, Campbell SF, Thornton JM: Molecular recognition. Conformational analysis of limited proteolytic sites and serine proteinase protein inhibitors. J Mol Biol 1991, 220:507-530.

34. Globularity of Proteins [http://bioinformatica.isa.cnr.it/GLOBU LARITY/]

Publish with Bio Med Central and every scientist can read your work free of charge

"BioMed Central will be the most significant development for disseminating the results of biomedical research in our lifetime. "

Sir Paul Nurse, Cancer Research UK

Your research papers will be:

- available free of charge to the entire biomedical community

- peer reviewed and published immediately upon acceptance

- cited in PubMed and archived on PubMed Central

- yours - you keep the copyright
BioMedcentral 